\title{
The glitch activity of rotation-powered pulsars
}

\author{
J. R. Fuentes ${ }^{1}$, C. M. Espinoza ${ }^{2}$ and A. Reisenegger ${ }^{1}$ \\ ${ }^{1}$ Instituto de Astrofísica, Pontificia Universidad Católica de Chile, Av. Vicuña Mackenna 4860, \\ 7820436, Santiago, Chile \\ ${ }^{2}$ Departamento de Física, Universidad de Santiago de Chile, Avenida Ecuador 3493, 9170124 \\ Estación Central, Santiago, Chile
}

\begin{abstract}
A statistical study of the glitch population and the behavior of the glitch activity across the known population of neutron stars is presented. A constant ratio between the glitch activity and the spin-down rate $\dot{\nu}_{\mathrm{g}} /|\dot{\nu}|=0.010 \pm 0.001$ is consistent with the behavior of all rotation-powered pulsars and magnetars. This relation is dominated by large glitches $(\Delta \nu \gtrsim$ $10 \mu \mathrm{Hz}$ ), which occur at a rate directly proportional to $|\dot{\nu}|$. The only exception are the rotationpowered pulsars with the highest values of $|\dot{\nu}|$, such as the Crab pulsar and PSR B0540-69, which exhibit a much smaller glitch activity, intrinsically different from each other and from the rest of the population. This contribution is based on the work done by Fuentes et al. (2017) "The glitch activity of neutron stars", accepted for publication in A\&A.
\end{abstract}

Keywords. stars: pulsars - stars: neutron - stars: magnetars - stars: rotation

\section{Introduction}

The rotation of neutron stars shows a very stable spin-down trend along time. Nevertheless, many neutron stars exhibit sudden increases in their rotation frequency $\nu$, known as glitches. Glitches have relative sizes $\Delta \nu / \nu \sim 10^{-11}-10^{-5}$, and in most cases, are followed by an increase in the spin-down rate $\dot{\nu}$ of the star. The physics behind glitches are not completely understood, but are believed to be caused by a neutron superfluid inside the star, which is rotating faster than the charged components of the star (Anderson \& Itoh 1975).

The observations of glitches are important since through them is possible to study indirectly the structural properties of neutron stars (Link et al. 1999; Pizzochero et al. 2017).

The continuous improving in timing techniques and long-term observations have allowed an increase in the number of glitches detected, and therefore, the possibility to observe different trends in the glitch behavior of neutron stars. McKenna \& Lyne (1990); Lyne et al. (2000); Espinoza et al. (2011) showed that the average spin-up rate of the star describes a linear behavior with the spin-down rate $|\dot{\nu}|$, and decreases with the characteristic age $\left(\tau_{c} \equiv \nu / 2|\dot{\nu}|\right)$, disappearing for objects with $\tau_{c}>20 \mathrm{Myr}$.

This contribution presents the main results of the statistical analysis of glitches in radio pulsars by Fuentes et al. (2017), in which the dependence of the glitch activity on long-term spin properties (spin frequency, spin-down rate, and combinations of these, such as energy loss rate, magnetic field, and spin-down age) was extensively studied.

\section{Glitch activity}

To quantify the cumulative effects of glitches on the rotation of a neutron star, we use the glitch activity parameter, defined as the time-averaged change of the rotation 
frequency due to glitches, in other words, "the spin-up rate". However, due to the short observation spans available for each star, it is not possible to detect enough glitches for a robust estimation of this parameter for individual pulsars. To avoid this problem, we studied the combined glitch activity for groups of neutron stars sharing a common property (we grouped the pulsars in bins of width equal to 0.5 in logarithmic scale). Following Lyne et al. (2000) and Espinoza et al. (2011), the average glitch activity for each group is

$$
\dot{\nu}_{\mathrm{g}}=\frac{\sum_{i} \sum_{j} \Delta \nu_{i j}}{\sum_{i} T_{i}},
$$

where the double sum runs over every change in frequency $\Delta \nu_{i j}$ due to the glitch $j$ of the pulsar $i$, and $T_{i}$ is the time over which pulsar $i$ has been searched for glitches. This analysis includes those pulsars that have been searched, but not found to glitch so far.

In this contribution we present the behavior of the activity as a function of the spindown rate $\dot{\nu}$ (see Fuentes et al. 2017 to see how the glitch activity correlates with the other parameters of neutron stars). We computed the glitch activity separately for large glitches $(\Delta \nu \geqslant 10 \mu \mathrm{Hz})$ and for small glitches (the remainder), showing the results in Figure 1 and Table 1 . The relation $\dot{\nu}_{\mathrm{g}} \propto|\dot{\nu}|$ already reported by Lyne et al. (2000) and Espinoza et al. (2011) for pulsars with $-14<\log |\dot{\nu}|<-10.5$ (we always take the units of $|\dot{\nu}|$ as $\mathrm{Hz} \mathrm{s}^{-1}$ ) is determined by large glitches. The mean value of the ratio $\dot{\nu}_{\mathrm{g}} /|\dot{\nu}|$ for this range is $0.012 \pm 0.001$. The activity due to small glitches follows a roughly similar, though noisier, increasing trend with $|\dot{\nu}|$.

\begin{tabular}{ccccccc}
\hline \hline \# bin & $\begin{array}{c}\log |\dot{\nu}| \\
\left(\mathrm{Hz} \mathrm{s}^{-1}\right)\end{array}$ & $\begin{array}{c}\sum T_{i} \\
(\mathrm{yr})\end{array}$ & $N_{\ell}$ & $N_{t}$ & $N_{\mathrm{pg}}$ & $N_{\mathrm{p}}$ \\
\hline 1 & -16.75 & 117 & 0 & 0 & 0 & 7 \\
2 & -16.25 & 430 & 0 & 0 & 0 & 25 \\
3 & -15.75 & 1233 & 0 & 0 & 0 & 70 \\
4 & -15.25 & 2478 & 0 & 3 & 3 & 139 \\
5 & -14.75 & 2675 & 0 & 11 & 8 & 142 \\
6 & -14.25 & 1973 & 0 & 25 & 16 & 105 \\
7 & -13.75 & 2083 & 0 & 35 & 20 & 113 \\
8 & -13.25 & 1706 & 1 & 29 & 18 & 105 \\
9 & -12.75 & 1312 & 3 & 26 & 14 & 81 \\
10 & -12.25 & 745 & 4 & 38 & 15 & 48 \\
11 & -11.75 & 493 & 8 & 74 & 15 & 33 \\
12 & -11.25 & 357 & 37 & 78 & 18 & 20 \\
13 & -10.75 & 66 & 13 & 19 & 5 & 5 \\
14 & -10.25 & 44 & 4 & 8 & 2 & 3 \\
15 & -9.75 & 16 & 0 & 2 & 1 & 1 \\
16 & -9.25 & 46 & 0 & 25 & 1 & 1 \\
\hline
\end{tabular}

Table 1. Statistics of glitches for pulsars binned by their spin-down rate. The first column is the bin number. The second and third columns correspond to $\log |\dot{\nu}|$ for the group of pulsars in each bin (the central value of each logarithmic interval), and the sum of the observation time of all pulsars in that bin. The next two columns contain the number of large glitches and the total number of glitches, respectively. The last two columns correspond to the number of pulsars with glitches, and the total number of pulsars in each bin, respectively.

Since the proportionality between the glitch activity and the spin-down rate is dominated by large glitches, and these have a very narrow size distribution, we expect that the rate of large glitches, $\dot{N}_{\ell}$, will also be proportional to $|\dot{\nu}|$. Because the number of large glitches is expected to follow a Poisson distribution, the expected dispersion in the rate of large glitches $\dot{N}_{\ell}$ can be estimated in a more reliable way than that in the glitch activity $\dot{\nu}_{\mathrm{g}}$. 


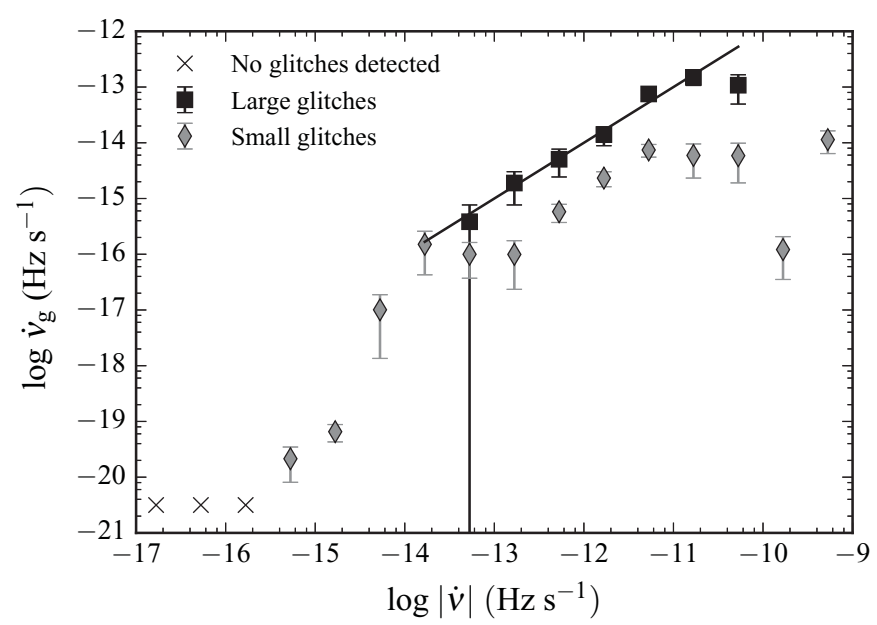

Figure 1. $\log \dot{\nu}_{\mathrm{g}}$ versus $\log |\dot{\nu}|$. The black squares and gray diamonds represent the glitch activity separately for large and small glitches, respectively. In both cases, $\dot{\nu}_{\mathrm{g}}$ was calculated for the same bins (groups) of pulsars, respectively. The straight line shows the linear relation $\dot{\nu}_{\mathrm{g}}=0.012|\dot{\nu}|$. The crosses denote bins with no detected glitches.

This allows us to test in a statistically meaningful way whether or not the identified trend applies to all pulsars.

Figure 2 confirms that $\dot{N}_{\ell} /|\dot{\nu}|$ is approximately constant and its mean value is $(4.2 \pm$ $0.5) \times 10^{2} \mathrm{~Hz}^{-1}$ (which we calculated considering only the $|\dot{\nu}|$ bins for pulsars with $-13.5<\log |\dot{\nu}|<-10.5)$. We observe that except for the three bins with the largest spin-down rate, all others are consistent with this trend. The non-detection of large glitches in the region of small $|\dot{\nu}|$ is consistent with the small expected rate and the finite monitoring time, as illustrated by the shaded area in Figure 2. Based on this relation, the expected number of large glitches $\left(N_{\ell}^{\exp }\right)$ for the three bins with the highest $|\dot{\nu}|$ $(\log |\dot{\nu}|>-10.5)$ is $30 \pm 5,35 \pm 5$, and $325 \pm 18$, respectively. This strongly contradicts the only four large glitches detected in bin 14 and the absence of large glitches in bins 15 and 16 (which contain only PSR B0540-69 and the Crab pulsar, respectively; see Table 1). Thus, we can confidently rule out the linear relation between $\dot{\nu}_{\mathrm{g}}$ and $|\dot{\nu}|$ for the largest values of the latter variable, but it remains consistent for all $|\dot{\nu}|<10^{-10.5} \mathrm{~Hz} \mathrm{~s}^{-1}$.

\section{Discussion}

We have shown that pulsars with $\dot{\nu}<10^{-10.5} \mathrm{~Hz} \mathrm{~s}^{-1}$ are consistent with a single trend, dominated by large glitches, in which the glitch activity $\dot{\nu}_{\mathrm{g}}$ is equal to $0.01|\dot{\nu}|$. The large collection of pulsars with no detected glitches is also consistent with this trend. For instance, the predicted rate of large glitches for pulsars with $\log |\dot{\nu}|=-14.25$ (bin 6 ) is one large glitch every $\sim 10^{4} \mathrm{yr}$, whereas the accumulated observing time in this bin is only $1973 \mathrm{yr}$ (Table 1), and this mismatch becomes even more extreme for the bins with lower $|\dot{\nu}|$. This means that there are no reasons to reject the idea that every neutron star will eventually experience a large glitch and will, in the long-term, follow the above relationship. On the other hand, we cannot rule out the possibility that, for example, the glitch mechanism could fail to produce large glitches in the pulsars with the smallest spin-down rates, or produce substantially more of them than predicted from the linear relation. Future studies, based on the analysis of glitches in individual pulsars, should provide information helping clarify this situation. For this, well designed, 


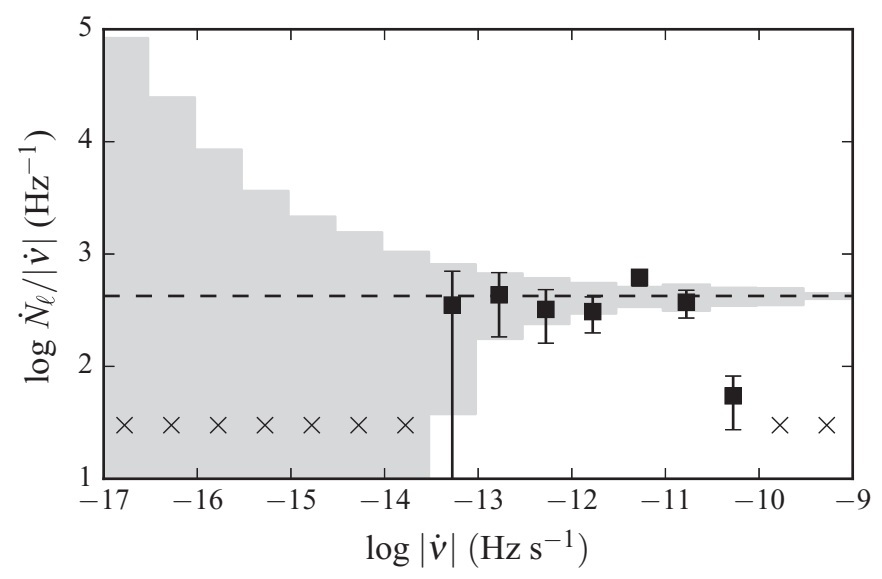

Figure 2. $\log \dot{N}_{\ell} /|\dot{\nu}|$ versus $\log |\dot{\nu}|$, where $\dot{N}_{\ell}$ is the number of large glitches per unit time. The horizontal line corresponds to the logarithm of the mean value $\left\langle\dot{N}_{\ell} /|\dot{\nu}|\right\rangle=(4.2 \pm 0.5) \times 10^{2} \mathrm{~Hz}^{-1}$, calculated over the bins with $-13.5<\log |\dot{\nu}|<-10.5$. The shaded region indicates the expected dispersion around this average value, based on a Poisson distribution of the number of large glitches and the available observing time spans. The black squares represent the observed values of the ratio $\dot{N}_{\ell} /|\dot{\nu}|$ for bins (groups) of pulsars. The crosses denote bins with no large glitches detected.

long-term monitoring campaigns are needed, in which cadence and sensitivity are combined to ensure the detection of small glitches, thereby improving the completeness of the current samples.

\section{References}

Anderson, P. W. \& Itoh, N. 1975, Nature, 256, 25

Espinoza, C. M., Lyne, A. G., Stappers, B. W., \& Kramer, M. 2011, MNRAS, 414, 1679

Lyne, A. G., Shemar, S. L., \& Smith, F. G. 2000, MNRAS, 315, 534

Link, B., Epstein, R. I., \& Lattimer, J. M. 1999, Phys. Rev. Lett., 83, 3362

McKenna, J. \& Lyne, A. G. 1990, Nature, 343, 349

Pizzochero, P. M., Antonelli, M., Haskell, B., \& Seveso, S. 2017, Nature Astronomy, 1, 0134

Espinoza, C. M., Lyne, A. G., \& Stappers, B. W. 2017, MNRAS, 466, 147

Lyne, A. G., Jordan, C. A., \& Graham-Smith, F. 2015, MNRAS, 446, 857 